\title{
$\mathrm{NO}_{3}$ 自由基与 $\mathrm{HNO}$ 及 $\mathrm{HONO}$ 反应机理研究
}

\author{
张吉东 ${ }^{a}$ 王海锋 ${ }^{*}, a$ 薛新英 ${ }^{a}$ 张岩文 ${ }^{a}$ 程新路 ${ }^{b}$ \\ ( ${ }^{a}$ 石河子大学 理学院物理系生态物理重点实验室 石河子 832003) \\ $\left({ }^{b}\right.$ 四川大学 原子与分子物理研究所 成都 610065)
}

\begin{abstract}
摘要 有研究显示 $\mathrm{NO}_{3}$ 自由基会在 $\mathrm{RDX}$ 热解过程中形成, 然而实验中却始终未有发现. 本文首次通过量子化学方法 研究了 $\mathrm{NO}_{3}$ 自由基分别与 $\mathrm{RDX}$ 两种热解产物 $\mathrm{HNO}$ 及 $\mathrm{HONO}$ 的反应，并利用 $\mathrm{CCSD}(\mathrm{T}) / 6-311++\mathrm{G}(\mathrm{d}, \mathrm{p})$ 方法对反应 势垒进行了精确计算. 计算结果显示, $\mathrm{NO}_{3}+\mathrm{HNO}$ 反应为无势垒反应, 且放出大量热量, 预示该反应极易发生. 这为 $\mathrm{NO}_{3}$ 自由基始终没有在 RDX 热解实验中被观察到提供了一种解释.
\end{abstract}

关键词 $\mathrm{NO}_{3}$; $\mathrm{HNO}$; $\mathrm{HONO}$; 反应机理

\section{Theoretical Study on the Reaction Mechanism of Nitrate Radical with HNO and HONO}

\author{
Zhang, Jidong $^{a} \quad$ Wang, Haifeng*,a $\quad$ Xue, Xinying $^{a} \quad$ Zhang, Yanwen $^{a} \quad$ Cheng, Xinlu $^{b}$ \\ ( ${ }^{a}$ Key Laboratory of Ecophysics and Department of Physics, School of Science, Shihezi University, Shihezi 832003) \\ ( ${ }^{b}$ Institute of Atomic and Molecular Physics, Sichuan University, Chengdu 610065)
}

\begin{abstract}
NO}_{3}$ radical is known as a dominant oxidant in the atmosphere at night, and plays an important role in atmospheric chemistry. Recently, $\mathrm{NO}_{3}$ radical is reported to be formed in the decomposition process of RDX, while it has never been detected in experiment up to now. The possible reason is that it would react with RDX or some RDX decomposition products once it is formed. This study for the first time theoretically investigated the bimolecular reactions of $\mathrm{NO}_{3}$ radical with two kinds of decomposition products of RDX (HNO and HONO) by computational chemistry methods, and the reaction mechanisms are studied using $\operatorname{CCSD}(\mathrm{T}) / 6-311++\mathrm{G}(\mathrm{d}, \mathrm{p})$ theoretical methods based on the geometric parameters optimized at the B3LYP/6-311++G(d,p) level. Same mechanisms are devised for the two reactions, such as oxygen abstraction and hydrogen abstraction. The two kinds of reaction pathways are of equal importance for the two reactions, because of similar barrier heights obtained in this study. The two pathways of the reation of $\mathrm{NO}_{3}+\mathrm{HNO}$ can result in two kinds of products $\mathrm{HNO}_{2}+\mathrm{NO}_{2}$ and $\mathrm{HNO}_{3}+\mathrm{NO}$ respectively, while the reaction of $\mathrm{NO}_{3}+\mathrm{HONO}$ only yields $\mathrm{HNO}_{3}+\mathrm{NO}_{2}$. The two reactions proceed with low barrier heights $(0 \sim 0.5 \mathrm{kcal} / \mathrm{mol}$, and $15.9 \sim 18.5 \mathrm{kcal} / \mathrm{mol}$ respectively), which are significantly lower than that of the reactions of $\mathrm{RDX}$ decomposition and $\mathrm{NO}_{3}$ formation. Moreover, the results show that the reaction of $\mathrm{NO}_{3}$ radical with HNO is barrierless and exothermic, implying it is energetically feasible to take place. The reaction may be responsible for the disappearance of $\mathrm{NO}_{3}$ radical during the decomposition process of RDX. Moreover, the isomerization reactions of $\mathrm{HNO}$ and HONO are also investigated by using the same methods, and a hydrogen atom intramolecular migration mechanism is found for these two isomerization reactions. The study is a necessary precursor for providing parameters for subsequent kinetic study and encourages further experimental investigation in future.
\end{abstract}

Keywords $\mathrm{NO}_{3}$; $\mathrm{HNO}$; $\mathrm{HONO}$; reaction mechanism

\section{1 引言}

$\mathrm{NO}_{3}$ 自由基是夜间大气中的重要氧化剂，由于其在 大气化学中所发挥的重要作用, 长期以来吸引了研究者 的广泛关注. 最近, Irikura 和 Johnson ${ }^{[1]}$ 报道, $\mathrm{NO}_{3}$ 自由基 会在 hexahydro-1,3,5-trinitro-1,3,5-triazine (RDX)的热分 解过程中形成, 因为 RDX 热解过程中主要分解产物之 一的 $\mathrm{NO}_{2}$ 分子会被 RDX 或者 RDX 的初始分解产物氧 化. RDX 是一种典型的硝胺类炸药, 也是当前研究最多 的单质炸药之一 ${ }^{[2-5]}$. 然而, 迄今为止 $\mathrm{NO}_{3}$ 自由基从未
在 RDX 热解实验中被发现 ${ }^{[1]}$, 究竟 $\mathrm{NO}_{3}$ 自由基能否在 RDX 热解过程中产生? 通常情况下, $\mathrm{NO}_{3}$ 自由基主要是 由 $\mathrm{NO}_{2}$ 分子氧化生成, 因此有理由相信, $\mathrm{NO}_{3}$ 自由基是 有可能会在 RDX 分解过程中形成的. 此外, Budzien 等 ${ }^{[6]}$ 通过分子动力学研究了 pentaerythritol tetranitrate (PETN) 的分解过程，也发现了 $\mathrm{NO}_{3}$ 自由基的产生. 然而, $\mathrm{NO}_{3}$ 自由基是如何在 RDX 的热解产物中消失的呢? 我们注 意到, $\mathrm{NO}_{3}$ 自由基具有很强的活性，以至于一旦形成就 可能与 RDX 或其热解产物反应而被消耗, 这在我们此 前的研究中也已被证实 ${ }^{[7]}$. 本文中我们将进一步研究

\footnotetext{
* E-mail: hfwang11@126.com

Received November 12; published December 6, 2012.

Project supported by the National Natural Science Foundation of China (No. 11145004).

项目受国家自然科学基金(No. 11145004)资助.
} 
$\mathrm{NO}_{3}$ 自由基与 $\mathrm{RDX}$ 热解中间体 $\mathrm{HNO}$ 及 $\mathrm{HONO}$ 之间的 反应，HONO 同时也是 HMX 等硝胺类炸药热解的重 要中间体之一[8,9].

多年来, 大量研究者一直致力于研究硝胺类炸药的 热分解过程 ${ }^{[8 \sim 12]}$, 但是由于其自身的复杂性, 硝铵类炸 药热解过程中所发生反应的化学机制却始终没有被完 全了解, 尤其是目前报道的大多数研究都仅局限于热解 过程中的单分子分解反应, 而热解过程中存在的双分子 反应却很少注意 ${ }^{[1]}$. 深刻理解炸药热解过程中所发生的 复杂化学过程对于炸药的研究是必不可少的, 因此本文 对 $\mathrm{NO}_{3}$ 自由基与 $\mathrm{HNO}$ 及 $\mathrm{HONO}$ 之间反应的研究将增进 对 RDX 热解过程双分子反应的理解, 并有助于今后对 硝铵类炸药热解过程的进一步研究.

\section{2 计算方法}

含能材料热解速度非常快，通过实验方法对反应进 行监测不易实现 ${ }^{[13]}$, 而量子化学方法(密度泛函 DFT)则 不仅具有方便、快捷、耗费资源较少的特点, 而且能够 提供可靠的准确性, 现在已经成为研究含能材料热解过 程中的化学反应所必备的工具 ${ }^{[14]}$. 本文主要采用密度 泛函理论对 $\mathrm{NO}_{3}$ 自由基与 $\mathrm{HNO}$ 及 $\mathrm{HONO}$ 反应机理进行 研究, 所涉及的所有计算都是通过 Gaussian 03 程序完 成的 ${ }^{[15]}$. 本文采用杂化密度泛函理论 B3LYP 方法 ${ }^{[16]}$ 在 6-311++ G(d,p)水平上对 $\mathrm{NO}_{3}$ 自由基与 $\mathrm{HNO}$ 及 $\mathrm{HONO}$ 反应过程中所有反应物、中间体、过渡态及产物的几何 构型进行了优化. 在相同理论水平上进行了频率计算, 以确定得到的驻点是最低点(稳定态, 没有虚频)还是鞍 点(过渡态, 仅有一个虚频), 并得到零点能(ZPE). 对得 到的反应过渡态均进行了内禀反应坐标(IRC) 跟踪计 算 ${ }^{[17]}$, 对反应势能面上过渡态与反应物、产物之间的连 接关系进行了验证. 本文采用 $\operatorname{CCSD}(\mathrm{T}) / 6-311++\mathrm{G}(\mathrm{d}, \mathrm{p})$ 方法 ${ }^{[18]}$ 对各驻点进行了单点能量计算. 本文中除特别
说明外，所涉及到的能量均包含零点能.

\section{3 结果与讨论}

\section{1 方法准确性判断}

为了评估本文所采用计算方法的准确性，我们将本 文计算得到的 $\mathrm{NO}_{3}$ 自由基的结构参数及振动频率与文 献报道的实验 ${ }^{[19]}$ 及理论值 ${ }^{[20]}$ 进行了比较, 见表 1 . 由表 1 可看出, 本文计算结果与已有实验值及理论值符合较 好，表明本文选用 B3LYP/6-311++G(d,p)方法进行结 构优化及频率计算是合适的.

表 $1 \mathrm{NO}_{3}$ 自由基键长 $(\AA)$ 、键角 $\left({ }^{\circ}\right)$ 及频率 $\left(\mathrm{cm}^{-1}\right)$ 与实验值和理论值的 对比

Table 1 The bond length $(\AA)$, bond angle $\left({ }^{\circ}\right)$, and vibrational frequencies $\left(\mathrm{cm}^{-1}\right)$ of $\mathrm{NO}_{3}$ with reported experimental and theoretical values

\begin{tabular}{ccccccccc}
\hline Ref. & N-O & O-N-O & $\omega_{1}$ & $\omega_{2}$ & $\omega_{3}$ & $\omega_{4}$ & $\omega_{5}$ & $\omega_{6}$ \\
\hline This & 1.234 & 120 & 283 & 284 & 800 & 1108 & 1108 & 1130 \\
work & & - & - & - & 762 & - & - & - \\
{$[19]$} & - & - & - & - & - & 1109 & 1109 & 1130 \\
\hline 20$]$ & 1.234 & 120 & 204 & 209 & 809 & 1106 &
\end{tabular}

\section{$3.2 \mathrm{NO}_{3}+\mathrm{HNO}$ 反应}

本文在 B3LYP/6-311++ G(d,p)水平上优化了 $\mathrm{NO}_{3}$ $+\mathrm{HNO}$ 反应各反应通道的反应物、中间体、过渡态及 产物的几何构型，优化得到的几何构型见图 1. 通过 $\operatorname{CCSD}(\mathrm{T}) / 6-311++\mathrm{G}(\mathrm{d}, \mathrm{p})$ 理论方法计算的各驻点能量 列于表 2. 图 2 中画出了该反应的反应势能面. 此反应 存在以下两个反应通道:

$$
\begin{aligned}
& \mathrm{NO}_{3}+\mathrm{HNO} \rightarrow \mathrm{HNO}_{2}+\mathrm{NO}_{2} \\
& \mathrm{NO}_{3}+\mathrm{HNO} \rightarrow \mathrm{HNO}_{3}+\mathrm{NO}
\end{aligned}
$$

接下来我们对各反应通道加以分析和讨论.

\section{2 .1 氧抽提反应}

$\mathrm{NO}_{3}+\mathrm{HNO}$ 反应的第一个通道是 $\mathrm{NO}_{3}$ 自由基中的
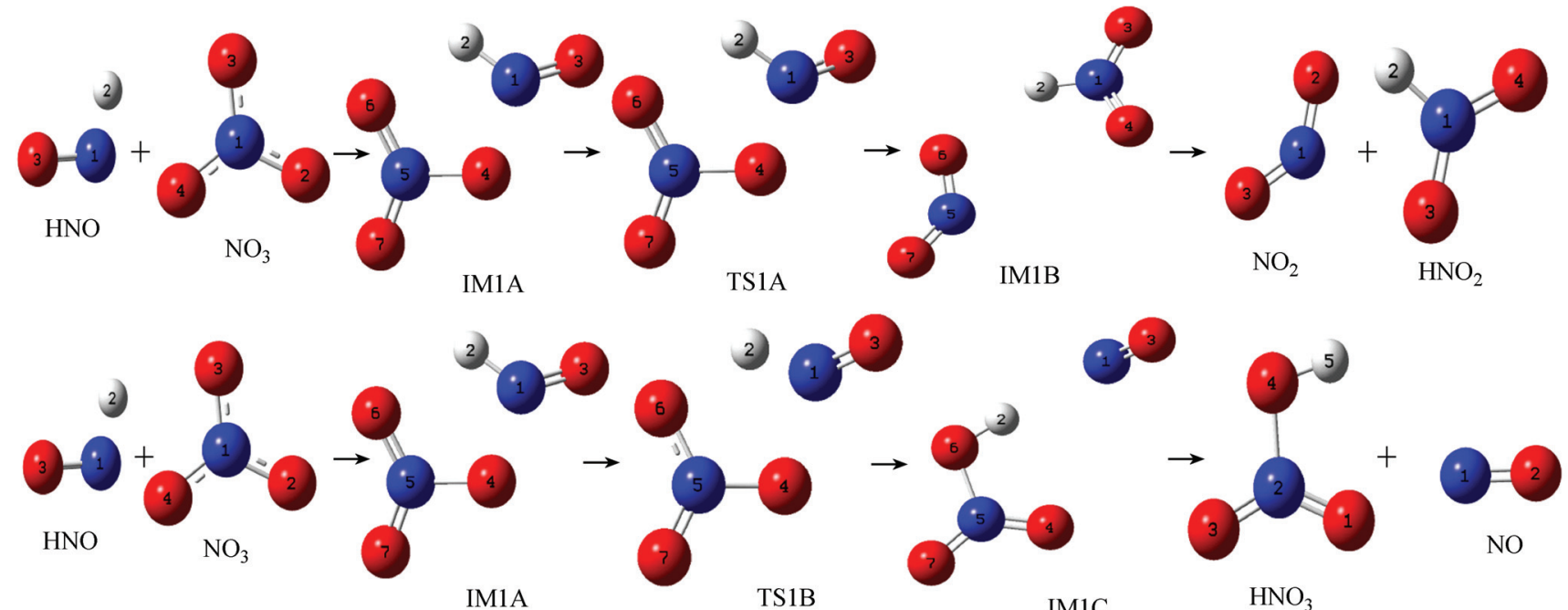

图 1 B3LYP/6-311++G(d,p)方法优化得到的 $\mathrm{NO}_{3}+\mathrm{HNO}$ 反应势能面上各驻点几何结构

Figure 1 B3LYP/6-311++G(d,p) geometries of minima and transition states associated with the reaction of $\mathrm{NO}_{3}+\mathrm{HNO}$ 


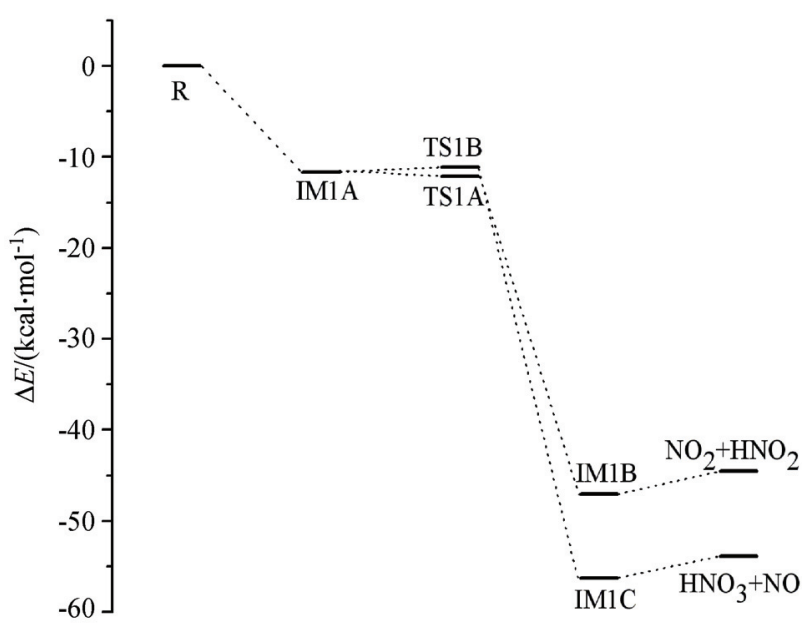

图 $2 \mathrm{CCSD}(\mathrm{T})$ 方法计算得到的 $\mathrm{NO}_{3}+\mathrm{HNO}$ 反应势能面

Figure 2 Potential energy diagram for the reaction of $\mathrm{NO}_{3}+\mathrm{HNO}$ obtained by $\operatorname{CCSD}(\mathrm{T})$

表 $2 \operatorname{CCSD}(\mathrm{T})$ 方法计算反应各驻点能量(含零点能)

Table 2 Molecular and transition-structure energies (including scaled ZPE) using $\operatorname{CCSD}(\mathrm{T})$

\begin{tabular}{cc||cc}
\hline Molecule & $\begin{array}{c}E_{0} / \text { hartree } \\
\text { CCSD }(\mathrm{T})\end{array}$ & Molecule & $\begin{array}{c}E_{0} / \text { hartree } \\
\mathrm{CCSD}(\mathrm{T})\end{array}$ \\
\hline $\mathrm{HNO}$ & -130.2107650 & $\mathrm{HNO}_{3}$ & -280.3279915 \\
$\mathrm{NO}_{3}$ & -279.6732618 & $\mathrm{NO}$ & -129.6419024 \\
$\mathrm{IM} 1 \mathrm{~A}$ & -409.9026209 & $\mathrm{HON}$ & -130.1426670 \\
$\mathrm{TS} 1 \mathrm{~A}$ & -409.9033650 & $\mathrm{TS} 1 \mathrm{C}$ & -130.0965680 \\
$\mathrm{IM} 1 \mathrm{~B}$ & -409.9589368 & $\mathrm{TS} 2 \mathrm{~A}$ & -205.2032144 \\
$\mathrm{NO}_{2}$ & -204.6758064 & $\mathrm{HONO}$ & -205.2938587 \\
$\mathrm{HNO}_{2}$ & -205.2791688 & TS2B & -484.9376491 \\
$\mathrm{TS}_{1 \mathrm{~B}}$ & -409.9017401 & TS2C & -484.9417177 \\
$\mathrm{IM} 1 \mathrm{C}$ & -409.9737216 & IM2A & -485.0084061 \\
\hline
\end{tabular}

$\mathrm{O}$ (4) 原子进攻 $\mathrm{HNO}$ 分子中的 $\mathrm{N}(1)$ 原子, 此时 $\mathrm{HNO}$ 分子 将从 $\mathrm{NO}_{3}$ 自由基中抽提 $\mathrm{O}$ 原子, 最终形成 $\mathrm{NO}_{2}+\mathrm{HNO}_{2}$. 反应开始后, $\mathrm{O}(4)$ 原子逐渐靠近 $\mathrm{N}(1)$ 原子而远离 $\mathrm{N}(5)$ 原 子, 从而形成中间体 IM1A, 如图 1 所示. IM1A 中, $\mathrm{N}(5)-\mathrm{O}(4)$ 键由 $\mathrm{NO}_{3}$ 自由基平衡结构的 $1.23 \AA$ 拉伸至 $1.40 \AA ; \mathrm{N}(1)-\mathrm{O}(4)$ 键正在形成, 键长为 $1.60 \AA$; 此外 IM1A 结构出现一氢键 $\mathrm{O}(6)-\mathrm{H}(2)$, 键长为 $1.95 \AA$. 从 图 2 可以看出, 生成 IM1A 时会放出 $11.7 \mathrm{kcal} / \mathrm{mol}$ 的热 量. 反应继续进行, 将形成过渡态 TS1A. 计算结果显示 在过渡态 TS1A 中, $\mathrm{N}(5)-\mathrm{O}(4)$ 键继续拉伸至 $1.43 \AA$, 而 $\mathrm{N}(1)-\mathrm{O}(4)$ 键长则缩短为 $1.53 \AA$. 本文利用 $\operatorname{CCSD}(\mathrm{T}) /$ 6-311 $++\mathrm{G}(\mathrm{d}, \mathrm{p})$ 方法对在 B3LYP/6-311+ $+\mathrm{G}(\mathrm{d}, \mathrm{p})$ 水平 上优化得到的过渡态的能量进行了计算. 计算显示, 过 渡态 TS1A 能量较中间体 IM1A 低 $0.4 \mathrm{kcal} / \mathrm{mol}$, 说明此 反应没有势垒. 这一数值明显低于 Scaldaferri 等 ${ }^{[20]}$ 报道 的 $\mathrm{H}_{2} \mathrm{~S}+\mathrm{NO}_{3}$ 反应的氧抽提反应势垒 $(24 \mathrm{kcal} / \mathrm{mol})$, 亦低 于此前我们研究组报道的 $\mathrm{TAZ}+\mathrm{NO}_{3}$ 反应的氧抽提反 应势垒 $(23.3 \mathrm{kcal} / \mathrm{mol})^{[7]}$. 为了验证这一结果的准确性, 我们分别通过 G3B3 组合方法 ${ }^{[21,22]}$ 和 $\operatorname{CCSD}(\mathrm{T}) /$ CC-pVDZ 方法对该反应势垒重新进行计算, 计算结果
分别为 -0.4 和 $-0.2 \mathrm{kcal} / \mathrm{mol}$, 三种方法计算结果非常 吻合，说明结果是可靠的. 所有计算结果均显示该通道 反应势垒接近 0 , 并且该反应在形成 IM1A 时放热明显, 说明该反应很容易进行. 随着反应的不断进行, $\mathrm{O}(4)$ 原 子继续靠近 $\mathrm{N}(1)$ 原子, 同时 $\mathrm{N}(5)-\mathrm{O}(4)$ 键被逐渐拉长, 直至形成中间体 IM1B. 在中间体 IM1B 中, $\mathrm{N}(1)-\mathrm{O}(4)$ 键长为 $1.219 \AA$, 与 $\mathrm{HNO}_{2}$ 平衡结构中 $\mathrm{NO}$ 键长 $1.216 \AA$ 非常接近; $\mathrm{N}(5)-\mathrm{O}(4)$ 键被拉长至 $3.57 \AA$, 即将断裂; 氢 键 $\mathrm{O}(6)-\mathrm{H}(2)$ 被拉伸至 $2.20 \AA$, 这预示着 IM1B 两部分 之间相互作用非常微弱. 从图 2 可看出 IM1B 只需吸收 $2.48 \mathrm{kcal} / \mathrm{mol}$ 能量即可形成最终的产物 $\mathrm{HNO}_{2}+\mathrm{NO}_{2}$. 此 通道中产物 $\mathrm{HNO}_{2}+\mathrm{NO}_{2}$ 能量较反应物 $\mathrm{NO}_{3}+\mathrm{HNO}$ 低 $44.5 \mathrm{kcal} / \mathrm{mol}$, 说明该反应通道是放热的.

\subsection{2 氢抽提反应}

$\mathrm{NO}_{3}+\mathrm{HNO}$ 反应的另一个通道是 $\mathrm{NO}_{3}$ 自由基中的 $\mathrm{O}(6)$ 原子进攻 $\mathrm{HNO}$ 分子中的 $\mathrm{H}(2)$ 原子, 此时 $\mathrm{NO}_{3}$ 自由 基将从 $\mathrm{HNO}$ 中抽提 $\mathrm{H}$ 原子, 最终形成 $\mathrm{HNO}_{3}+\mathrm{NO}$. 反 应开始后，与氧抽提反应相同，先形成中间体 IM1A. 此后 $\mathrm{H}(2)$ 原子逐渐靠近 $\mathrm{O}(6)$ 原子而远离 $\mathrm{N}(1)$ 原子，从而 形成过渡态 TS1B. TS1B 是类反应物型过渡态, 如图 1 所示过渡态 TS1B 中, $\mathrm{N}(1)-\mathrm{H}(2)$ 键仅比 $\mathrm{HNO}$ 平衡结构 中的 $\mathrm{N}-\mathrm{H}$ 键长 $0.01 \AA$, 而 $\mathrm{N}(5)-\mathrm{O}(6)$ 键也仅由 $\mathrm{NO}_{3}$ 自 由基平衡结构中 $\mathrm{N}-\mathrm{O}$ 键拉伸 $0.02 \AA, \mathrm{O}(6)-\mathrm{H}(2)$ 键正 在形成, 键长为 $1.66 \AA$. 频率分析显示过渡态 TS1B 仅 有一个虚频, 大小为 $255 \mathrm{i} \mathrm{cm}^{-1}$. 从图 2 可看出该反应势 垒为 $0.5 \mathrm{kcal} / \mathrm{mol}$, 而该通道生成中间体 IM1A 过程所放 热量足可使反应顺利进行，所以该反应也很容易进行. 此通道与氧抽提反应相比势垒略高, 与 Scaldaferri 等 ${ }^{[20]}$ 报道的 $\mathrm{H}_{2} \mathrm{~S}+\mathrm{NO}_{3}$ 反应的氢抽提反应势垒 $0 \sim 0.8$ $\mathrm{kcal} / \mathrm{mol}$ 相当. 随着反应的不断进行, $\mathrm{H}(2)$ 原子继续靠近 $\mathrm{O}(6)$ 原子, 同时 $\mathrm{N}(1)-\mathrm{H}(2)$ 键被逐渐拉长, 直至形成中 间体 IM1C. 在中间体 IM1C 中, $\mathrm{O}(6)-\mathrm{H}(2)$ 键缩短至 $0.978 \AA$, 与 $\mathrm{HNO}_{3}$ 平衡结构中 $\mathrm{O}-\mathrm{H}$ 键长 $0.972 \AA$ 非常 接近; $\mathrm{N}(5)-\mathrm{O}(6)$ 键被拉长至 $1.407 \AA$, 与 $\mathrm{HNO}_{3}$ 平衡结 构中 $\mathrm{N}-\mathrm{OH}$ 键长 $1.416 \AA$ 非常接近; 此外, 氢键 $\mathrm{N}(1)$ $-\mathrm{H}(2)$ 距离已达 $2.14 \AA$, 即将断裂. 图 2 显示 IM1C 只需 $2.4 \mathrm{kcal} / \mathrm{mol}$ 能量即可分裂成最终的产物 $\mathrm{HNO}_{3}+\mathrm{NO}$, 且此通道放热 $53.9 \mathrm{kcal} / \mathrm{mol}$.

\subsubsection{HNO 异构化反应}

我们在对 HNO 分子结构进行优化时, 发现了 $\mathrm{HNO}$ 的异构体 HON, 结构如图 3 所示. 能量计算发现 HON 较 HNO 能量高 $42.7 \mathrm{kcal} / \mathrm{mol}$, 因此 HNO 比 HON 结构 稳定. 我们设计了 HNO 分子的异构化反应，该反应可 通过氢原子由氧原子到氮原子的迁移实现. HON 中 $\mathrm{O}(3)-\mathrm{H}(2)$ 键长为 $1.00 \AA$, 反应开始后氢原子由氧原子 向氮原子迁移, 形成过渡态 $\mathrm{TS} 1 \mathrm{C}$ 后, $\mathrm{O}(3)-\mathrm{H}(2)$ 键被拉 伸至 $1.11 \AA$, 而 $\mathrm{N}(1)-\mathrm{H}(2)$ 键逐渐形成. 频率分析显示 过渡态 TS1C 仅有一个虚频, 大小为 $2228 \mathrm{~cm}^{-1}$. 反应继 
续进行, 最终形成 HNO. 此反应势垒为 $28.9 \mathrm{kcal} / \mathrm{mol}$, 且放热 $42.7 \mathrm{kcal} / \mathrm{mol}$, 所以反应较易进行, 而反向反应 势垒较高为 $71.6 \mathrm{kcal} / \mathrm{mol}$, 所以较难发生.

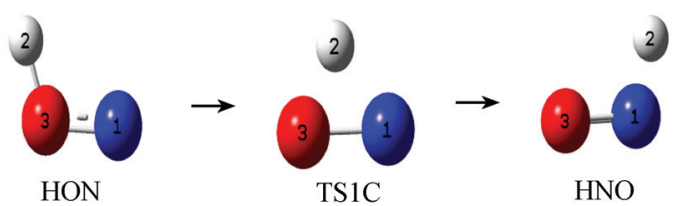

图 3 B3LYP/6-311++G(d,p)方法优化得到的 HNO 异构化反应势能 面上各驻点几何结构.

Figure 3 B3LYP/6-311 + $+\mathrm{G}(\mathrm{d}, \mathrm{p})$ geometries of minima and transition states associated with the isomerization reaction of $\mathrm{HNO}$

\section{3 $\mathrm{NO}_{3}+\mathrm{HONO}$ 反应}

在此部分中, 我们研究了 $\mathrm{HONO}$ 异构化反应及 $\mathrm{NO}_{3}$ $+\mathrm{HONO}$ 反应. HONO 异构化反应的几何构型见图 4. $\mathrm{NO}_{3}+\mathrm{HONO}$ 反应与 $\mathrm{NO}_{3}+\mathrm{HNO}$ 反应一样, 也存在氧抽 提与氢抽提两个反应通道, 两反应通道的产物均为 $\mathrm{HNO}_{3}+\mathrm{NO}_{2} . \mathrm{NO}_{3}+\mathrm{HONO}$ 各反应通道的反应物、中间 体、过渡态及产物的几何构型见图 5, 反应势能面见图 6.

\subsubsection{HONO 异构化反应}

对 $\mathrm{HNO}_{2}$ 结构进行优化, 发现了两种不同的稳定结 构. 一种是 $\mathrm{NO}_{3}+\mathrm{HNO}$ 反应氧抽提通道生成的 $\mathrm{HNO}_{2}$, 另一种是 HONO, 其几何结构如图 4 所示. HONO 是 RDX 和 HMX 热解的初始产物 ${ }^{[8,9]}$, 计算显示 $\mathrm{HONO}$ 能 量较 $\mathrm{HNO}_{2}$ 低 $9.2 \mathrm{kcal} / \mathrm{mol}$, 因此 $\mathrm{HONO}$ 结构较 $\mathrm{HNO}_{2}$ 更稳定. 本文设计了 $\mathrm{HNO}_{2}$ 分子的异构化反应, 该反应 亦可通过氢原子由氧原子到氮原子的迁移实现. $\mathrm{HNO}_{2}$ 中 $\mathrm{N}(1)-\mathrm{H}(2)$ 键长为 $1.04 \AA$, 反应开始后氢原子由氮原 子向氧原子迁移, 形成过渡态 TS $2 \mathrm{~A}$ 后, $\mathrm{N}(1)-\mathrm{H}(2)$ 键被 拉伸至 $1.19 \AA$, 而即将形成的 $\mathrm{O}(4)-\mathrm{H}(2)$ 键长为 $1.30 \AA$.

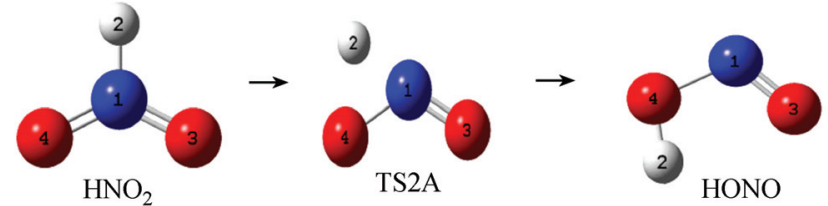

图 4 B3LYP/6-311++G(d,p)方法优化得到的 HONO 异构化反应势 能面上各驻点几何结构.

Figure 4 B3LYP/6-311 $++\mathrm{G}(\mathrm{d}, \mathrm{p})$ geometries of minima and transition states associated with the isomerization reaction of $\mathrm{HONO}$

过渡态 TS2A 仅有一个虚频, 大小为 $2074 \mathrm{i} \mathrm{cm}^{-1}$, 通过 对虚频振动模式分析, 该过渡态确实连接 $\mathrm{HNO}_{2}$ 与 HONO. $\mathrm{H}(2)$ 原子进一步向 $\mathrm{O}(4)$ 原子靠近, 最终形成 HONO. 该反应势垒为 $47.6 \mathrm{kcal} / \mathrm{mol}$, 较 $\mathrm{HON}$ 异构化反 应高，而反向反应势垒稍高为 $56.9 \mathrm{kcal} / \mathrm{mol}$.

\section{3 .2 氧抽提反应}

$\mathrm{NO}_{3}+\mathrm{HONO}$ 反应的第一个通道是 $\mathrm{NO}_{3}$ 自由基中的 $\mathrm{O}(5)$ 原子进攻 $\mathrm{HONO}$ 分子中的 $\mathrm{N}(1)$ 原子, 此时 $\mathrm{HONO}$ 分子将从 $\mathrm{NO}_{3}$ 自由基中抽提 $\mathrm{O}$ 原子, 最终形成 $\mathrm{NO}_{2}+$ $\mathrm{HNO}_{3}$. 反应开始后, $\mathrm{O}(5)$ 原子逐渐靠近 $\mathrm{N}(1)$ 原子而远离 $\mathrm{N}(6)$ 原子, 直至形成过渡态 TS2B. 如图 5 所示, TS2B 中, $\mathrm{N}(6)-\mathrm{O}(5)$ 键由 $\mathrm{NO}_{3}$ 自由基平衡结构的 $1.23 \AA$ 被显著拉 伸至 $1.41 \AA ; \mathrm{N}(1)-\mathrm{O}(5)$ 键正在形成, 键长为 $1.69 \AA$; 此 外, HONO 部分中的 $\mathrm{O}(2)-\mathrm{N}(1)-\mathrm{O}(4)$ 键角由平衡结构 中的 $113.8^{\circ}$ 增大至 $119.5^{\circ}$. 频率分析显示过渡态 TS2B 仅有一个虚频, 大小为 $262 \mathrm{i} \mathrm{cm}^{-1}$. 本文利用 $\operatorname{CCSD}(\mathrm{T})$ 方 法对这一过渡态能量进行了计算, 得到该反应势垒高度 为 $18.5 \mathrm{kcal} / \mathrm{mol}$, 这一势垒与 Scaldaferri 等 ${ }^{[20]}$ 报道的 $\mathrm{H}_{2} \mathrm{~S}+\mathrm{NO}_{3}$ 反应的氧抽提反应势垒 $24 \mathrm{kcal} / \mathrm{mol}$ 稍低, 亦 低于此前我们研究组报道的 $\mathrm{TAZ}+\mathrm{NO}_{3}$ 反应的氧抽提 反应势垒 $23.3 \mathrm{kcal} / \mathrm{mol}^{[7]}$, 但明显高于 $\mathrm{NO}_{3}+\mathrm{HNO}$ 反应
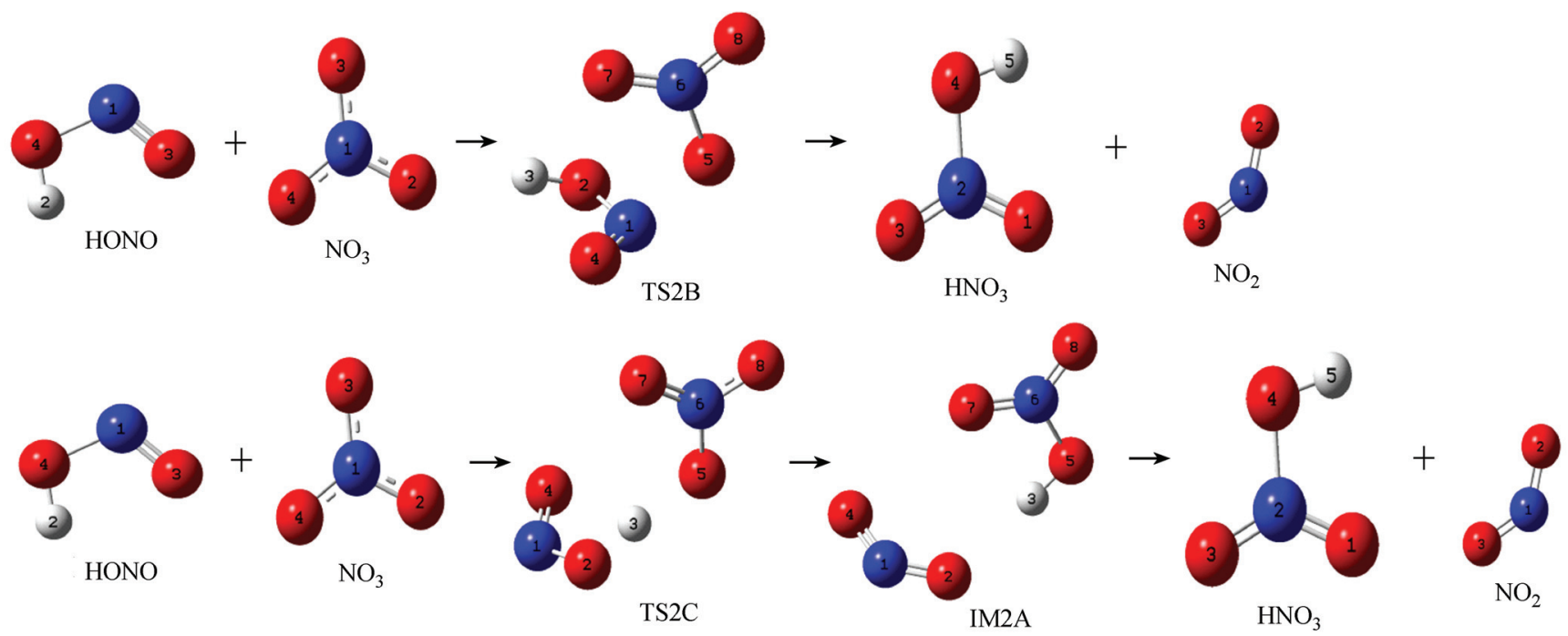

图 5 B3LYP/6-311++ G(d,p) 方法优化得到的 $\mathrm{NO}_{3}+\mathrm{HONO}$ 反应势能面上各驻点几何结构

Figure 5 B3LYP/6-311++G(d,p) geometries of minima and transition states associated with the reaction of $\mathrm{NO}_{3}+\mathrm{HONO}$ 


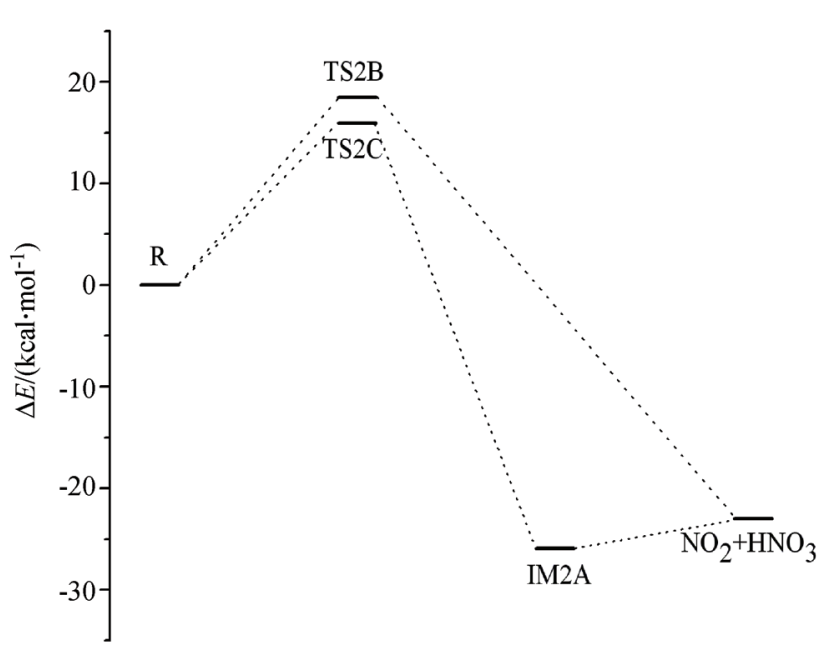

图 $6 \mathrm{CCSD}(\mathrm{T})$ 方法计算得到的 $\mathrm{NO}_{3}+\mathrm{HONO}$ 反应势能面

Figure 6 Potential energy diagram for the reaction of $\mathrm{NO}_{3}+\mathrm{HONO}$ obtained by $\operatorname{CCSD}(\mathrm{T})$

的氧抽提反应势垒. 随着反应的不断进行, $\mathrm{O}(5)$ 原子继 续靠近 $\mathrm{N}(1)$ 原子, 同时 $\mathrm{N}(6)-\mathrm{O}(5)$ 键被继续拉伸直至断 裂, 最终形成产物 $\mathrm{NO}_{2}+\mathrm{HNO}_{3}$. 该通道放热 23.0 $\mathrm{kcal} / \mathrm{mol}$, 放出的热量可提供反应进行所需能量, 反应 较易进行.

\subsection{3 氢抽提反应}

$\mathrm{NO}_{3}+\mathrm{HONO}$ 反应的另一个通道是 $\mathrm{NO}_{3}$ 自由基中的 $\mathrm{O}(5)$ 原子直接进攻 $\mathrm{HONO}$ 分子中的 $\mathrm{H}(3)$ 原子, 此时 $\mathrm{NO}_{3}$ 自由基将从 $\mathrm{HONO}$ 中抽提 $\mathrm{H}$ 原子, 最终形成 $\mathrm{HNO}_{3}+$ $\mathrm{NO}_{2}$. 反应开始后, $\mathrm{H}(3)$ 原子逐渐靠近 $\mathrm{O}(5)$ 原子而远离 $\mathrm{O}(2)$ 原子, 从而形成过渡态 TS2C. 如图 5 所示过渡态 $\mathrm{TS} 2 \mathrm{C}$ 中, $\mathrm{O}(2)-\mathrm{H}(3)$ 键被拉伸至 $1.06 \AA ; \mathrm{N}(6)-\mathrm{O}(5)$ 键 由 $\mathrm{NO}_{3}$ 自由基平衡结构中 $\mathrm{N}-\mathrm{O}$ 键长 $1.23 \AA$ 拉伸至 1.30 $\AA$; 而 $\mathrm{O}(5)-\mathrm{H}(3)$ 键正在形成, 键长为 $1.45 \AA$. 从图 6 可 看出该反应势垒为 $15.9 \mathrm{kcal} / \mathrm{mol}$. 此通道与氧抽提反应 相比势垒略低, 但高于 $\mathrm{NO}_{3}+\mathrm{HNO}$ 反应的氢抽提通道 势垒, 也高于 Scaldaferri 等 ${ }^{[20]}$ 报道的 $\mathrm{H}_{2} \mathrm{~S}+\mathrm{NO}_{3}$ 反应的 氢抽提反应势垒. 随着反应的不断进行, $\mathrm{H}(3)$ 原子继续 靠近 $\mathrm{O}(5)$ 原子, 同时 $\mathrm{O}(2)-\mathrm{H}(3)$ 键被逐渐拉长, 直至形 成中间体 IM2A. 在中间体 IM2A 中, $\mathrm{O}(5)-\mathrm{H}(3)$ 键缩短 至 $0.976 \AA$, 与 $\mathrm{HNO}_{3}$ 平衡结构中 $\mathrm{O}-\mathrm{H}$ 键长 $0.972 \AA$ 非 常接近; $\mathrm{N}(6)-\mathrm{O}(5)$ 键被拉长至 $1.41 \AA$, 非常接近 $\mathrm{HNO}_{3}$ 平衡结构中 $\mathrm{N}-\mathrm{OH}$ 键长 $1.42 \AA ; \mathrm{O}(2)-\mathrm{N}(1)-\mathrm{O}(4)$ 键角 由 $\mathrm{HONO}$ 平衡结构中的 $113.8^{\circ}$ 增大至 $134.5^{\circ}$, 与 $\mathrm{NO}_{2}$ 分子平衡结构的 $134.4^{\circ}$ 非常接近; 此外, 氢键 $\mathrm{O}(2)-$ $\mathrm{H}(3)$ 距离已达 $2.05 \AA$, 即将断裂. 图 6 显示 IM2A 只需 $2.9 \mathrm{kcal} / \mathrm{mol}$ 能量即可分裂成最终的产物 $\mathrm{HNO}_{3}+\mathrm{NO}_{2}$, 且此通道放热 $23.0 \mathrm{kcal} / \mathrm{mol}$.

总的来看, $\mathrm{NO}_{3}+\mathrm{HNO}$ 反应与 $\mathrm{NO}_{3}+\mathrm{HONO}$ 反应机 理相同, 都存在氧抽提和氢抽提反应. $\mathrm{NO}_{3}+\mathrm{HNO}$ 反应 的氧抽提通道没有势垒, 而氢抽提通道势垒也仅为 0.55 $\mathrm{kcal} / \mathrm{mol}$, 考虑到计算方法本身存在的误差, 这两种通
道对于 $\mathrm{NO}_{3}+\mathrm{HNO}$ 反应同样重要. $\mathrm{NO}_{3}+\mathrm{HONO}$ 反应中 氧抽提和氢抽提反应势垒也相差不大, 分别为 18.5 及 $15.9 \mathrm{kcal} / \mathrm{mol}$, 因此在反应中应同时存在这两种通道. 这与 Scaldaferri等 ${ }^{[20]}$ 报道的 $\mathrm{H}_{2} \mathrm{~S}+\mathrm{NO}_{3}$ 反应不同, 在 $\mathrm{H}_{2} \mathrm{~S}$ $+\mathrm{NO}_{3}$ 反应中, $\mathrm{H}_{2} \mathrm{~S}+\mathrm{NO}_{3}$ 反应的氢抽提通道反应势垒为 $0 \sim 0.8 \mathrm{kcal} / \mathrm{mol}$, 而氧抽提通道反应势垒为 $24 \mathrm{kcal} / \mathrm{mol}$, 因此氢抽提反应较氧抽提反应更易发生, 是 $\mathrm{H}_{2} \mathrm{~S}+\mathrm{NO}_{3}$ 反应的主要通道. 此外, 标题反应的势垒远低于已报道 的 RDX 初始分解反应势垒 $(39.0 \mathrm{kcal} / \mathrm{mol})^{[23]}$ 及 $\mathrm{NO}_{3}$ 自由 基生成反应势垒 $(32.5 \mathrm{kcal} / \mathrm{mol})^{[1]}$, 并且反应产物也都在 RDX 热解产物中存在, 说明标题反应是完全有可能在 $\mathrm{RDX}$ 热分解过程中发生的. 而 $\mathrm{NO}_{3}+\mathrm{HNO}$ 反应势垒接 近为 0 且放热, 说明该反应极易发生, 可解释 $\mathrm{NO}_{3}$ 自由 基在 RDX 热解过程中生成, 但在实验中却没有发现的 问题.

\section{4 结论}

本文对 $\mathrm{NO}_{3}+\mathrm{HNO}$ 反应及 $\mathrm{NO}_{3}+\mathrm{HONO}$ 反应机理 进行了研究, 得出如下结论:

(1) 标题反应均存在氧抽提和氢抽提通道, 且两个 通道反应势垒相差不大, 因此同样重要.

(2) 标题反应势垒远低于已报道的 RDX 初始分解 反应及 $\mathrm{NO}_{3}$ 自由基生成反应势垒，预示标题反应完全有 可能在 RDX 热分解过程中发生.

(3) $\mathrm{NO}_{3}+\mathrm{HNO}$ 反应势垒接近为 0 且放热，说明该 反应极易发生，可解释为什么 $\mathrm{NO}_{3}$ 自由基在 $\mathrm{RDX}$ 热解 过程中生成, 但在实验中却没有发现.

\section{References}

[1] Irikura, K. K.; Johnson, R. D. J. Phys. Chem. A 2006, 110, 13974.

[2] Li, M. M.; Shen, R. Q.; Li, F. S. Acta Phys.-Chim. Sin. 2011, 27(6), 1379. (李苗苗, 沈瑞琪, 李风生, 物理化学学报, 2011, 27(6), 1379.)

[3] Niu, X. Q.; Zhang, J. G.; Feng, X. J.; Chen, P. W.; Zhang, T. L.; Wang, S. Y.; Zhang, S. W.; Zhou, Z. N.; Yang, L. Acta Chim. Sinica 2011，69(14), 1627. (牛晓庆, 张建国, 冯晓军, 陈鹏万, 张同来, 王世英, 张绍文, 周遵宁, 杨利, 化学学报, 2011, 69(14), 1627.)

[4] Goldberg, I. G.; Swift, J. A. Cryst. Growth Des. 2012, $12(2), 1040$.

[5] Patterson, J. E.; Dreger, Z. A.; Gupta, Y. M. J. Phys. Chem. B 2007, $111,10897$.

[6] Budzien, J.; Thompson, A. P.; Zybin, S. V. J. Phys. Chem. B 2009, 113,13142 .

[7] Zhang, J. D.; Cheng, X. L.; Zhao, F. Propellants, Explos., Pyrotech. 2010, 35, 315.

[8] Chakraborty, D.; Muller, R. P.; Dasgupta, S.; Goddard, W. A. J. Phys. Chem. A 2001, 105, 1302.

[9] Zhang, S. W.; Nguyen, H. N.; Truong, T. N. J. Phys. Chem. A 2003, $107,2981$.

[10] Isayev, O.; Gorb, L.; Qasim, M.; Leszczynski, J. J. Phys. Chem. B 2008, 112, 11005

[11] Wang, G. X.; Gong, X. D.; Liu, Y.; Du, H. C.; Xu, X. J.; Xiao, H. M. J. Hazard. Mater. 2010, 177, 703.

[12] Zhang, J. G.; Wang, K.; Niu, X. Q.; Zhang, S. W.; Feng, X. J.; Zhang, T. L.; Zhou, Z. N. J. Mol. Model. 2012, 18, 3915.

[13] Jadhav, H. S.; Talawar, M. B.; Sivabalan, R.; Dhavale, D. D.; Asthana, S. N. J. Hazard. Mater. 2007, 143, 192.

[14] Su, X. F.; Cheng, X. L.; Meng, C. M.; Yuan, X. L. J. Hazard. Mater 2009, 161, 551 .

[15] Frisch, M. J.; Trucks, G. W.; Schlegel, H. B.; Scuseria, G. E.; Robb, 
M. A.; Cheeseman, J. R.; Montgomery, J. A.; Jr., Vreven, T.; Kudin, K. N.; Burant, J. C.; Millam, J. M.; Iyengar, S. S.; Tomasi, J.; Barone, V.; Mennucci, B.; Cossi, M.; Scalmani, G.; Rega, N.; Petersson, G. A.; Nakatsuji, H.; Hada, M.; Ehara, M.; Toyota, K.; Fukuda, R.; Hasegawa, J.; Ishida, M.; Nakajima, T.; Honda, Y.; $\quad$ Kitao, O.; Nakai, H.; Klene, M.; Li, X.; Knox, J. E.; Hratchian, H. P.; Cross, J. B.; Adamo, C.; Jaramillo, J.; Gomperts, R.; Stratmann, R. E.; Yazyev, O.; Austin, A. J.; Cammi, R.; Pomelli, C.; Ochterski, J. W.; Ayala, P. Y.; Morokuma, K.; Voth, G. A.; Salvador, P.; Dannenberg, J. J.; Zakrzewski, V. G.; Dapprich, S.; Daniels, A. D.; Strain, M. C.; Farkas, O.; Malick, D. K.; Rabuck, A. D.; Raghavachari, K.; Foresman, J. B.; Ortiz, J. V.; Cui, Q.; Baboul, A. G.; Clifford, S.; Cioslowski, J.; Stefanov, B. B.; Liu, G.; Liashenko, A.; Piskorz, P.; Komaromi, I.; Martin, R. L.; Fox, D. J.; Keith, T.; Al-Laham, M. A.; Peng, C. Y.; Nanayakkara, A.; Challacombe, M.; Gill, P. M. W.;
Johnson,B.; Chen, W.; Wong, M. W.; Gonzalez, C.; Pople, J. A. Gaussian 03, Revision A.1, Gaussian, Inc., Pittsburgh, PA, 2003.

[16] Becke, A. D. J. Chem. Phys. 1993, 98, 5648.

[17] Gonzalez, C.; Schlegel, H. B. J. Phys. Chem. 1990, 94, 5523.

[18] Raghavachari, K.; Trucks, G. W.; Pople, J. A.; Head-Gordon, M. Chem. Phys. Lett. 1989, 157, 479.

[19] Friedl, R. R.; Sander, S. P. J. Phys. Chem. 1987, 91, 2721.

[20] Scaldaferri, M. C. L.; Pimentel, A. S. Chem. Phys. Lett. 2009, 470, 203.

[21] Curtiss, L. A.; Raghavachari, K.; Redfern, P. C.; Rassolov, V.; Pople, J. A. J. Chem. Phys. 1998, 109, 7764.

[22] Baboul, A. G.; Curtiss, L. A.; Redfern, P. C.; Raghavachari, K. J. Chem. Phys. 1999, 110, 7650.

[23] Chakraborty, D.; Muller, R. P.; Dasgupta, S.; Goddard, W. A. J. Phys. Chem. A 2000, 104, 2261.

(Cheng, B.) 